\title{
The Evaluation of Node Importance in Urban Road Network Based on Complex Network Theory
}

\author{
Hongmei Cao ${ }^{1, \mathrm{a}}$, Hao Liu ${ }^{1}$, Fang Zhao ${ }^{1}$, Yuanyuan $\mathrm{Li}^{1}$ and Mingkun $\mathrm{Du}^{1}$ \\ ${ }^{1}$ Inner Mongolia university, China
}

\begin{abstract}
Identifying and protecting traffic hub is vital to keep the stability and reliability of urban road traffic network. In this paper, a comprehensive evaluation method of node importance in urban road traffic network was introduced based on identification theory of key nodes in complex network. Firstly, taking into account the impact of road network topology and traffic flow characteristics on node's capacity, node importance in topological network and betweenness, urban road grade and node traffic flow are selected as the evaluating indexes of node importance, and AHP is used to determine the weight of each index. Secondly, the associated value of the indexes are calculated and obtained based on SuperMap Deskpro and practical investigation, and then, combined with TOPSIS, a sequence of node importance is determined. Finally, we applied the method to part district of Hohhot road traffic network, it can be seen that the key nodes obtained with the method stay in the important position in the the traffic network and have greater traffic flow, the results showed it is effective and practical feasible.
\end{abstract}

\section{Introduction}

Complex networks are highly abstract of complex systems which often have a large number of nodes[1]. The studies of network stability have shown that as long as $5 \%$ of the key nodes being attacked the network will be basically paralyze[2]. The applications of the research on node importance in real life are very meaningful. For example, we can identify the key character and focus fire against him in the terrorist organization network[3], and the congestion problem can be solved by finding the nodes with the most flow in a communication network[4].

The research on the evaluation of node importance in urban road traffic network which is a kind of complex and integrated network is also very pregnant. Wang Wei proposed that key node is refer to the node that is crucial to overall characteristic of network, and its damage will cause the loss of the function of network, simultaneously make the network connectivity, reliability and the road network performance drop rapidly[5]. We can infer that the protection of the vital nodes in the urban traffic network has great significance to ease the congestion situation, improve the invulnerability ability and enhance the reliability of urban road traffic network.

In order to explore the method of identifying the vital nodes in urban road traffic network, this paper presented a kind of method of evaluating comprehensively the node importance of urban road traffic network. And this method will be introduced in the following.

\section{Selection of indexes}

\subsection{Selection of network topological characteristic indexes}

Domestic methods of evaluation of node importance in complex network have been relatively mature. Chen Yong[6] and Tan[7] evaluated node importance by means of node deletion and node constriction. Since the two methods have no local analysis on the nodes, the final results are not accurate or convincing. Then many scholars further studied the methods of evaluating node importance in complex network. Zhou put the node contribution and node efficiency as the considering factors[1]. A method which is based on node connectivity and node criticality is presented[8]. Qin and Yang listed six indexes, and seven of them are finally selected for the evaluation by principal component analysis[9] (PCA).

When complex network theory is applied to the urban traffic network, it must be concerned that the degree of the nodes in urban road traffic network is much too single because of the existence of intersections and t-junctions. So some above methods are not suitable for the research on road network, for example it is not suitable for Qin and Yang's method to apply to the evaluation of node importance in urban road network, because degree of the nodes in urban road network is mostly 3 or 4 . In addition, considering 
the lengths of the roads between the intersections make a great difference, the inter-node length can be concerned in the process of selecting indexes.

The paper chose node importance in topological network and betweenness as network topological characteristic indexes. This paper will recommend them as follows.

Node importance in topological network $\left(C_{i}\right)$ is the product of the node efficiency $\left(I_{i}\right)$ and the sum of the importance contribution $\left(H_{i}\right)$ of its adjacent nodes, as given by Eq. (1), Eq. (2) and Eq. (3).

$$
\begin{gathered}
C_{i}=I_{i} \times H_{i} \\
H_{i}=\sum_{j=1, j \neq i}^{n} \delta_{i j} D_{j} I_{j} /<k>^{2} \\
I_{j}=\frac{1}{n} \sum_{m=1, m \neq j}^{n} \frac{1}{d_{j m}}
\end{gathered}
$$

Where $\delta_{i j}$ is the adjacency coefficient, $D_{j}$ is the degree of node $v_{j}, d_{j m}$ is the distance between node $v_{j}$ and node $v_{j}$, and $k$ is the average degree of the selected network.

Node betweenness $\left(B_{i}\right)$ refers to the number of shortest paths which pass node $v_{j}$ between all the pairs of nodes in the network, was calculated as Eq. (4).

$$
I_{j}=\frac{1}{n} \sum_{m=1, m \neq j}^{n} \frac{1}{d_{j m}}
$$

Where $n_{j k}$ is the number of the shortest paths between node $v_{j}$ and node $v_{k}$, and $n_{j k}(i)$ is the number of the shortest paths which pass node $v_{i}$ between node $v_{j}$ and node $v_{k}$.

\subsection{Selection of traffic flow characteristic indexes}

It has just started in recent years that complex network theory was applied to the research on node importance of urban road traffic network, the literature of this aspect is not a lot. Wang screened out six indexes including delay, queue length and degree of saturation from eleven indexes by AHP[4], although the results obtained by this method is accurate, it is difficulty to get the indexes and it would cost huge manpower resources to evaluate large networks. Zhong proposed a kind of weighted method of the nodes base on urban road grade, and calculated the node importance according to the node traffic flow and topology value which is got by connection degree and weights of nodes[10].

This paper chose node traffic flow and urban road grade as the traffic flow characteristic indexes. Traffic flow of the intersections can intuitively reflect the significance of the intersections in urban road network. And the significance of the intersections consist of the roads in different grade differs from each other. Stated below .
In the light of the status, traffic function and serve function of urban roads, the roads can be divided into four categories: expressway $(k)$, major arterial $(z)$, secondary arterial $(c)$ and collector $(g)$. The quantitative analysis of urban road grade can be conducted on the basis of Table 1. For example, the weight the intersection consist of a expressway and a mayor arterial is: $\mathrm{G}_{\mathrm{i}}=2 \times 10+2 \times 8=36$. The weight is the value used in conducting quantitative analysis of urban road grade in this paper.

Table 1. Calculation method of node weight[10]

\begin{tabular}{lcccc} 
Road & Expressway & Major arterial & Secondary arterial & Collector \\
\hline Weight & 10 & 8 & 5 & 3 \\
\hline
\end{tabular}

We define the node traffic flow as the sum of traffic flow on the roads which connect the node, as given by Eq. (5).

$$
Q i=\sum_{n=1}^{m} f(i, n)
$$

Where $m$ is the number of the nodes that are connected to node $i$ and $f(i, n)$ is the traffic flow which is in the direction of entering node $i$ on the road which connect node $n$ and $i$.

\section{Evaluation procedure}

The evaluation is conducted around data collection and data processing. The specific steps of the evaluation as follows:

(1) Load urban road network diagram into the software SuperMap Deskpro, then get the urban road network topological diagram by using the function of topological data processing of the software.

(2) Calculate the node importance in topological network by the functions of adjacency point analysis and measuring the distance of SuperMap Deskpro, then analysis and calculate node betweenness by the function of best path analysis of the software. Finally get the data about node traffic flow and urban road grade by investigation, searching the Internet and inquiring the traffic administration.

(3) Determine indexes weights by analytic hierarchy process(AHP). Firstly, determine the target layer, criterion layer and index layer, then set up hierarchical analysis model, as shown in Figure 1. Secondly, establish judgment matrix by 1 9 method in Table 2 . Finally calculate the weights of indexes according to the judgment matrix and verify conformance.

(4) Calculate and sort the node importance by using TOPSIS. Firstly, establish a date matrix which has been normalized. Secondly, find the most ideal solution $Z^{+}$and the least ideal solution $Z^{-}$, then calculate the distance respectively from each node to $Z^{+}$and $Z^{-}$, and record the result as $D^{+}$and $D^{-}$. It needs to be stressed that this calculation has to take 
into account the weights obtained above. Thirdly, figure up the node importance by Eq. (6). Finally, Sort node importance.

$$
E_{i}=\frac{D^{+}}{D^{+}+D^{-}}
$$

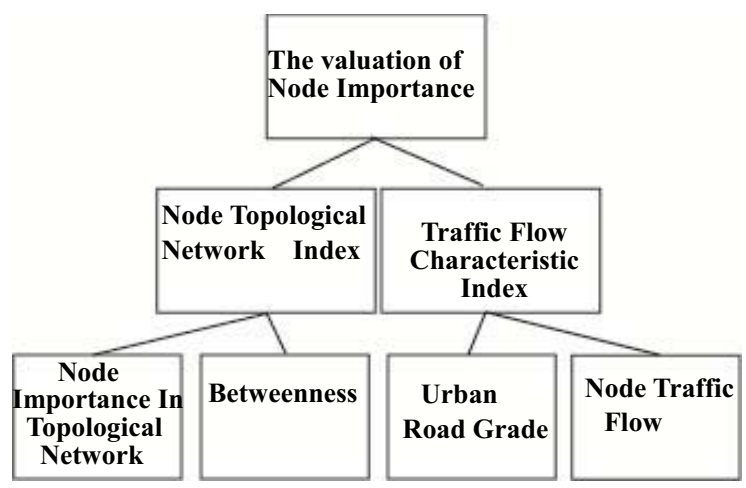

Figure 1. Hierarchical analysis mode

Table 2. 1 9 scale method

\begin{tabular}{cl}
\hline Scale & \multicolumn{1}{c}{ Meaning } \\
\hline 1 & Two factors are equally important \\
3 & The former is more important than the latter \\
5 & The former is more important than the latter obviously \\
7 & The former is more important than the latter strongly \\
9 & The former is more important than the latter extremely \\
$2,4,6,8$ & The intermediate value between adjacent judgments \\
Reciprocal & The importance of scale of the latter than the former \\
\hline
\end{tabular}

\section{The case study on Hohhot urban road traffic network}

\subsection{Selection of the study area and the nodes}

The planning and construction of urban road traffic network get increasingly the attention of people with the speeding up of urbanization process, and the traffic management puts a high value on the revamping of traffic trunk network. This paper selected the study area which encircled by West Second Ring Road, South Second Ring Road, West XinHua Street, South TongDao Road, DaBei Street, DaNan Street and ZhaoJun Road which are showed in Figure 2. And the topological network diagram can be obtained by SuperMap Deskpro. It needs to be added that the paper abstracted the roads into non directional edges, and the branches with small traffic flow, narrow alleys and streets, as well as the small intersections without traffic lights are neglected in the process of topology operation. In the topological network, this paper selected sixteen nodes as the research objects, and labeled them as Figure 3. Their specific composition is as shown in Table 3.

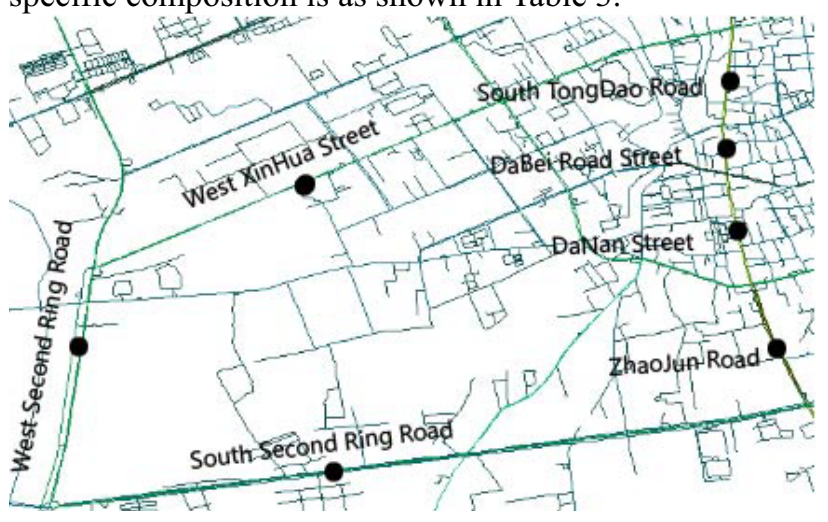

Figure 2. Network diagram of part district of Hohhot road traffic network

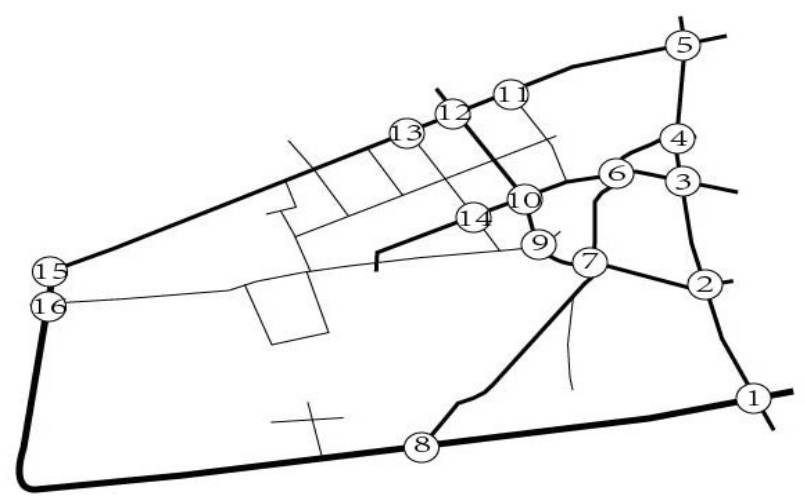

Figure 3. Serial number of the selected nodes Table 3. List of nodes

\begin{tabular}{cc}
\hline Node & Node composition \\
\hline 1 & ZhaoJun Road-South Second Ring Road \\
2 & DaNan Street-Ordos Street \\
3 & DaBei Street-DaNan Street-DaDong Street-DaXi Street \\
4 & South TongDao Road-DaBei Street-XiShun City Street-West \\
& ZhongShan Road \\
5 & South TongDao Road-North TongDao Road-XinHua \\
& Street-West XinHua Road \\
6 & XiShun City Street-XianFu Street-DaXi Street \\
8 & XiShun City Street-YunZhong Road-Ordos Street \\
9 & South Second Ring Road-YunZhong Road \\
10 & South Bayinnaoer Road-Ordos Street-West Ordos Street \\
11 & South Bayinnaoer Road-XianFu Street \\
12 & West XinHua Road-South AJiLaQin Road \\
& South Bayinnaoer road-North Bayinnaoer road-West \\
13 & XinHua Street \\
14 & North ALaShan Road-West XinHua Street \\
15 & South ALaShan Road-XianFu Street \\
16 & GuangMing Street-West Second Ring Road \\
& West Ordos Street-West XinHua Road \\
\hline
\end{tabular}

\subsection{Data preparation}

The steps of getting the date are as follows, and the date is listed in Table 4. 
Table 4. The list of date

\begin{tabular}{|c|cccc|}
\hline Node & $\begin{array}{c}\text { Topological network } \\
\text { importanceofnode }\left(\times 10^{-4}\right)\end{array}$ & Betweenness & $\begin{array}{c}\text { Node traffic } \\
\text { flow }\end{array}$ & $\begin{array}{c}\text { Weighted value of } \\
\text { the node }\end{array}$ \\
\hline 1 & 2.7 & 0 & 6072 & 32 \\
2. & 12.5 & 2 & 5124 & 32 \\
3 & 29.4 & 6 & 5016 & 32 \\
4 & 25.5 & 9 & 6144 & 32 \\
5 & 9.2 & 1 & 7692 & 32 \\
6 & 37.2 & 25 & 5280 & 32 \\
7 & 11.3 & 26 & 4280 & 32 \\
8 & 3.3 & 0 & 4586 & 32 \\
9 & 29.9 & 27 & 3912 & 26 \\
10 & 41.6 & 29 & 3564 & 32 \\
11 & 23.8 & 4 & 5904 & 24 \\
12 & 29.6 & 14 & 3340 & 32 \\
13 & 20.5 & 8 & 2928 & 24 \\
14 & 23.6 & 11 & 3264 & 26 \\
15 & 14.5 & 4 & 4376 & 24 \\
16 & 17.7 & 9 & 3664 & 26 \\
\hline
\end{tabular}

(1) Calculate node importance in topological network. The node importance can be got by above step 2 and the definition of node topological importance.

(2) Calculate the betweenness. The betweenness would be got by using the analysis function of optimal path in SuperMap Deskpro.

(3) Count node traffic flow. This paper recorded the peak hour flow of the nodes as the node traffic flow by on-the-spot investigation.

(4) Conduct quantitative analysis of the nodes considering the urban road grade. The Quantitative analysis can be conducted by the method in 2.2 .

\subsection{Determination of indexes weights}

The weights of the indexes can be determined by using AHP, and the weights obtained are as given in Table 5 .

Table 5. Weights of the indexes

\begin{tabular}{ccccc}
\hline Index & $\begin{array}{c}\text { Node importance in } \\
\text { Topological network }\end{array}$ & Betweenness & $\begin{array}{c}\text { Node traffic } \\
\text { flow }\end{array}$ & $\begin{array}{c}\text { Urban road } \\
\text { grade }\end{array}$ \\
\hline Weight & 0.375 & 0.125 & 0.100 & 0.400 \\
\hline
\end{tabular}

\subsection{Sorting of nodes importance}

This paper calculated the node importance by TOPSIS, the orders are as Table 6.

It can be seen that node 6,10 and 9 are the first three in the ranking of node importance from Table 6 and node 6 is the first. The first three nodes are ranked respectively in second, first and third place in node topological network importance and forth, first and second place in betweenness, they are the hinges for the vehicles which connected to the north and south, east and west. On the other hand, there is more traffic flow in node 6 than node 10 and 9, that is why node 6 is most important. If node 6 is removed, many of the vehicles in the network will have to bypass and it will affect traffic efficiency of the vehicles. After the analysis, the nodes obtained by the method can be consistent well with the actual situation.
Table 6. Sorting of nodes importance

\begin{tabular}{clc}
\hline Ranking & Node & Node importance \\
\hline 1 & 6 & 0.247 \\
2 & 10 & 0.317 \\
3 & 9 & 0.388 \\
4 & 4 & 0.478 \\
5 & 3 & 0.502 \\
6 & 12 & 0.503 \\
7 & 11 & 0.551 \\
8 & 7 & 0.564 \\
9 & 14 & 0.573 \\
10 & 5 & 0.624 \\
11 & 2 & 0.660 \\
12 & 13 & 0.674 \\
13 & 16 & 0.687 \\
14 & 15 & 0.743 \\
15 & 1 & 0.748 \\
16 & 8 & 0.847 \\
\hline
\end{tabular}

\section{Summary}

An evaluation method of node importance in urban road traffic network was introduced based on identification theory of key nodes in complex network in the paper. This paper tried to avoid lengthy evaluation process under the premise of making the results comprehensive and accurate. The nodes obtained by this method are in good agreement with the reality compared with the actual urban road network. In the future research on the evaluation, scholars will try to make the evaluation process more concise and the evaluation results more accurate. Through the research is getting harder, there is no doubt that the research is bound to promote the development of urban road traffic better.

\section{References}

1. X. Zhou, Finding vital node by node importance evaluation matrix in Complex networks[J]. Acta Physica Sinica, 61(5): 201-208. (2012)

2. X. Wang, Y. Ling, YL. Fei, Personalization recommendation system based on web log\&cache data mining[J]. Journal of the China Society for Scientific And Technical Information, 24(3): 324-328. (2005)

3. S. Haykin, Cognitive radio: Brain-empowered wireless communi-Cations[J]. IEEE Journal on Selected Areas in Communications, 23(2): 201-220. (2005)

4. FX. B, J. Budazik, K.J. Hammond. Mining navigation history for recommendation[C]. Proc of the International Conf on Intelligent User Interfaces, ACM. New Orleans. LA, ACM, 106-112. (2000)

5. W. Wang, Research on the method of key node and section distinguish of urban rode traffic network. Jilin University. (2015)

6. Y. Chen, AQ. Hu, X. Hu. China Institute Commun, 25, 129 (2004) 
7. YJ. Tan , J. Wu, HZ. Deng, Syst. Eng. Theory \& Practice. 26, 79 (2006)

8. J. Chen, LF. Sun. Evahiation of node importance in complex networks[J]. Journal of Souzhwest Jiaotong University, 44 (3): 426-429. (2009)

9. L. Qin, ZL. Yang, Synthesis evaluation method for node importance in complex networks. Computer Science, 42 (2): 60-63. (2015)

10. R. Zhong, Research of key nodes and important sections of the road network[D]. BUPT. (2013) 